\title{
Supraclavicular lymph node metastasis in elderly patients undergoing esophageal squamous cell carcinoma radical surgery: construction of risk and prognostic predictive nomograms
}

\author{
Ling Chen ${ }^{1,2 *}$, Shaobin $\mathrm{Yu}^{1,2 *}$, Xiaohong Jiang ${ }^{3}$, Mingqiang Kang ${ }^{1,2}$ \\ ${ }^{1}$ Department of Thoracic Surgery, Fujian Medical University Union Hospital, Fuzhou, China; ${ }^{2}$ Key Laboratory of Ministry of Education for \\ Gastrointestinal Cancer; Fujian Key Laboratory of Tumor Microbiology, Fujian Medical University, Fuzhou, China; ${ }^{3}$ Fuzhou Center for Disease \\ Control and Prevention, Fuzhou, China \\ Contributions: (I) Conception and design: L Chen; (II) Administrative support: M Kang; (III) Provision of study materials or patients: L Chen, M \\ Kang; (IV) Collection and assembly of data: L Chen, S Yu, X Jiang; (V) Data analysis and interpretation: L Chen, S Yu; (VI) Manuscript writing: All \\ authors; (VII) Final approval of manuscript: All authors. \\ "These authors contributed equally to this work. \\ Correspondence to: Mingqiang Kang, MD, PHD. Director of Department Administration, Chief Physician, Department of Thoracic Surgery, Fujian \\ Medical University Union Hospital, 29 Xinquan Road, Fuzhou 350000, China. Email: mingqiangkang@126.com.
}

Background: Supraclavicular lymph node metastasis (SCLN) is an adverse prognostic determinant of esophageal cancer. However, lymphadenectomy for SCLN is a traumatic procedure, especially in elderly patients, which is associated with more postoperative complications. Currently, identification of risk factors of SCLN metastasis and avoidance of unnecessary lymphadenectomy for SCLN in esophageal squamous cell carcinoma (ESCC) patients has become an unmet clinical need.

Methods: A total of 90 elderly patients with ESCC between January 2008 and December 2013 was eligible for this analysis. Logistic regression was performed to determine risk factors for SCLN metastasis after ESCC radical surgery in elderly patients. A nomogram was constructed to individually predict the risk for SCLN metastasis. The Kaplan-Meier survival curve and cumulative risk curve were further analyzed to evaluate the effect of SCLN metastasis after ESCC radical surgery on survival prognosis and cumulative risk assessment in elderly patients. Finally, the SCLN metastasis group and the independent risk factor group were fitted by drawing a decision curve to evaluate the net benefit of the model.

Results: SCLN developed in 38 patients $(42.2 \%)$. Postoperative lymph node metastasis $(\mathrm{P}<0.05)$, tumor thrombus $(\mathrm{P}<0.05)$ and tumor infiltration $(\mathrm{P}<0.05)$ were independent risk factors for SCLN metastasis. The influence of SCLN metastasis on postoperative survival in elderly ESCC patients was statistically significant $(\mathrm{P}=0.028, \mathrm{P}<0.05)$; with the passage of time, the cumulative risk of SCLN metastasis increased, the survival probability decreased, and the survival time was shortened.

Conclusions: Postoperative lymph node metastasis, tumor thrombus and tumor infiltration are independent risk factors for recurrence and metastasis of SCLNs in elderly patients with esophageal squamous cell carcinoma. The nomogram model based on these factors provides a preliminary reference for individualized risk assessment, prognosis guidance and decision-making of SCLN metastasis in elderly patients with esophageal squamous cell carcinoma (ESCC).

Keywords: Elderly; esophageal squamous cell carcinoma (ESCC); lymph node metastasis; nomogram; decision curve

Submitted Mar 18, 2020. Accepted for publication Oct 19, 2020.

doi: $10.21037 /$ jtd-20-1388

View this article at: http://dx.doi.org/10.21037/jtd-20-1388

(C) Journal of Thoracic Disease. All rights reserved. 


\section{Introduction}

In China, the incidence of esophageal squamous cell carcinoma (ESCC) is 20.9 out of 100,000, which is the highest in the world (1-3). At the time of ESCC treatment, most patients are in the middle and late stages, and there are a large number of patients with supraclavicular lymph node (SCLN) metastasis (10.3-17.6\%). Lymph node surgery and radical surgery are considered to be the main diagnostic treatments for patients with resectable $\operatorname{ESCC}(4,5)$. While there have been no strict prospective, randomized, controlled clinical studies to provide supporting evidence, with the use of a lymph node cleaning scope, the greater the surgical effect is, and the better the radical cure effect; however, the operation risk will also increase, especially in the neck, such as with chest minor cleaning for cervical anastomotic fistula, resulting in laryngeal recurrent nerve injury and a high incidence of respiratory complications, affecting the patients' rapid recovery and quality of life (6-8). Therefore, how to reasonably choose the range of lymph node dissection for patients with high risk of SCLN metastasis, and to ensure the curative effect while reducing the adverse reactions of surgery is the current problem.

In recent years, there are some "selective minor lymph node cleaning" studies $(9,10)$ performed, aiming at providing radical surgery, reducing operation risks, improving survival, and making treatment accuracy more robust. However, these studies have some limitations. First, even if preoperative SCLN metastasis was found and immediate salvage treatment was implemented, there was still a lag in treatment. Second, the risk of SCLN metastasis was not objectively quantified, and assessments based on subjective experience are not rigorous enough.

By searching multiple databases, including PubMed, the nomogram model has been widely and successfully used for prediction and survival analyses of a variety of cancers, quantifying risks by considering all known clinical variables, thus allowing individualized risk assessment and prognosis prediction of a variety of cancers (11-18). However, a prediction model that can be applied for the clinical quantitative assessment $(19,20)$ of SCLN metastasis in terms of the risks of ESCC surgery has not been established. Therefore, in this paper, a nomogram prediction model was established to analyze the known clinical data to quantify SCLN metastasis risk assessment in the postoperative period, aiming to target elderly patients with ESCC (twofield lymphadenectomy) if further retrospective radiation and chemotherapy or SCLN cleaning is needed after surgery and to provide an important reference for treatment decisions.

We present the following article in accordance with the TRIPOD reporting checklist (available at http://dx.doi. org/10.21037/jtd-20-1388).

\section{Methods}

\section{Ethics statement}

The study was conducted in accordance with the Declaration of Helsinki (as revised in 2013). The study was approved by institutional/regional/national ethics/ committee/ethics board of Fujian Union hospital (No.: 2020KY017) and informed consent was taken from all the patients. The study did not constitute harm and potential risks to donors.

\section{Patients}

Between January 1 of 2008 and December 31 of 2013, we collected data for 409 patients who underwent esophagectomy for ESCC at the Fujian Union Hospital, China. Surgeries were performed by experienced surgeons of the Fujian Union Hospital. A total of 90 eligible patients were enrolled in this study according to the following criteria.

Inclusion criteria: (I) age was $\geq 65$ (21); (II) patients had radical esophagectomy [Ivor-Lewis esophagectomy (22), McKeown esophagectomy and left thoracic esophagectomy] and standard radical dissection of the two-field thoracic and abdominal lymph nodes; (III) preoperative imaging examination suggested a ct $1-3 \mathrm{n} 0 \mathrm{~m} 0$ stage tumor and no SCLN enlargement; (IV) postoperative follow-up indicated the presence of SCLN enlargement, and puncture indicated metastasis; (V) preoperative ECOG physical condition score was $0-1$; (VI) preoperative ASA score was I-III; and (VII) informed consent was obtained from the patients.

Exclusion criteria: (I) severe mental illness; (II) preoperative imaging examination suggested enlarged SCLNs; (III) 5-year history of other malignancies, including lymphatic and hematologic malignancies; (IV) patients with esophageal cancer who had received preoperative neoadjuvant chemoradiotherapy; (V) a history of unstable angina or myocardial infarction within 5 or 6 months; (VI) a history of cerebral infarction or cerebral hemorrhage within 6 or 6 months; (VII) a history of continuous systemic corticosteroid therapy within 1 month; (XI) simultaneous 
surgical treatment for other diseases was required; $(\mathrm{X})$ pulmonary function FEV1 < estimated value 50\%.

Rejection criteria: (I) cases confirmed as M1 intraoperative/postoperative: no evidence of distant metastasis was found in the preoperative examination, while distant metastasis was confirmed from intraoperative exploration/postoperative pathology; (II) intraoperative/ postoperative T4b stage was confirmed; (III) it was proven intraoperatively that regional lymph node fusion into clusters could not ensure R0 resection or the resectability of wrapping the important vessels; (IV) simultaneous surgical treatment of other diseases was required; (V) after inclusion, due to preoperative sudden severe complications (unable to tolerate surgery or anesthesia) the treatment plan of this study was not suitable or could not be implemented as planned.

The collected clinical data included sex, age, smoking history, drinking history, CEA, operation mode and consistent approach, preoperative SCLNs color to exceed, postoperative follow-up SCLNs color to exceed result, SCLN biopsy results after surgery, preoperative imaging stage, tumor location, tumor length, the total number of lymph nodes cleaned, the number of positive lymph nodes, lymph node metastasis, tumor differentiation, the presence of vascular tumors, tumor infiltration, tumor TNM staging, T stage, $\mathrm{N}$ stage, $\mathrm{M}$ in installment, follow-up time, death, survival time, and time of death.

\section{Follow-up}

Follow-up was conducted every three months for the first three years, every six months for the next two years, and once a year after five years. Follow-up of the study was complete in the May of 2015, and achieved a minimal follow-up duration of 18 months in the data set. Overall survival (OS) was measured from the time of surgery to the last date of follow-up. The survival status of patients was collected at the last date of follow-up.

\section{Statistical analysis}

SPSS 21. xdowns software was used for the statistical evaluation (SPSS, Chicago, Illinois, USA) as well as r 3.1.2 software (Institute of Statistics and Mathematics, Vienna, Austria). Logistic analysis was performed using univariate analysis to determine the risk factors for SCLN metastasis after ESCC radical surgery; logistic multifactor analysis was again used to determine the independent risk factors for SCLN metastasis and the area under the ROC curve by ROC curve drawing and calculating the AUC for singlefactor and multi-factor analysis for the prediction value and the forecasting accuracy. The following were also computed: the fit of the Hosmer-Lemeshow goodness-of-fit $(\mathrm{H})$ evaluation model. Nomogram of the map projections, logistic regression model, and risk quantification of SCLN metastasis in terms of the postoperative risk for individualized evaluation, combined with each independent risk factor on the top of the scale to determine the point of the corresponding values. Then, the total score was determined on the overall scale to identify the probability of risk prediction of SCLN metastasis in ESCC patients after the operation for the first time. With COX, singlefactor and multi-factor analyses were used to determine the independent risk factors for the development of SCLN metastasis; and with logarithmic rank and inspection, SCLN metastasis was assessed in elderly ESCC patients after radical surgery in terms of Kaplan-Meier survival curve test variables and cumulative risk curves. These tests were carried out to evaluate the influence of SCLN metastasis on the prognosis of survival and cumulative risk assessment. Finally, a decision curve was drawn to fit the SCLN metastasis group and multiple independent risk factor group for evaluating the net benefit of the model.

$\mathrm{P}<0.05$ was considered statistically significant. All statistical analyses were performed using SPSS21 for MAC (Chicago, Illinois) and RStudio - 1.2.1335 (http:// www.r-project). Hmisc, grid, lattice, Formula, ggplot2, survminer, RMS, survival, peperr, rmda and mass package were used.

\section{Results}

\section{Clinical features}

Among the 90 patients, 30 were female (33.33\%), and 60 were male $(66.67 \%)$. The average age was 70.44 years old (65-85 years old). Ten elderly patients $(11.11 \%)$ had positive postoperative lymph node metastasis (two-field thoracic and abdominal lymph nodes), vascular tumor thrombus and infiltration tissue, and the SCLN metastasis rate was $100 \%(10 / 10)$, while 41 elderly patients $(45.56 \%)$ had negative postoperative lymph node metastasis (twofield thoracic and abdominal lymph nodes), vascular tumor thrombus and infiltration tissue, and the SCLN metastasis rate was $9.76 \%$. 
Table 1 Logistic univariate analysis of radical treatment for supraclavicular lymph node metastasis in elderly ESCC patients

\begin{tabular}{|c|c|c|c|c|}
\hline Variable & No. of patients & $\%$ & $\mathrm{P}$ & $95.0 \% \mathrm{Cl}$ for $\operatorname{Exp}(\mathrm{B})$ \\
\hline Male & 60 & 66.7 & & \\
\hline Female & 30 & 33.3 & & \\
\hline Age (years) & & & 0.953 & $0.093-6.847$ \\
\hline Positive & 25 & 27.8 & & \\
\hline Negative & 65 & 72.2 & & \\
\hline Vascular tumor thromboembolism & & & $<0.001$ & $5.739-367.040$ \\
\hline Positive & 19 & 21.1 & & \\
\hline Positive & 38 & 42.2 & & \\
\hline Negative & 52 & 57.8 & & \\
\hline Tumor location & & & 0.834 & $1.970-2.006$ \\
\hline Upper & 15 & 16.7 & & \\
\hline Middle & 59 & 65.6 & & \\
\hline Lower & 16 & 17.7 & & \\
\hline T stage (post-operatively) & & & 0.127 & $2.707-2.904$ \\
\hline $\mathrm{T} 1$ & 15 & 16.7 & & \\
\hline No & 55 & 61.1 & & \\
\hline $\mathrm{N} 1$ & 23 & 25.6 & & \\
\hline N2 & 12 & 13.3 & & \\
\hline M stage (post-operatively) & & & 0.142 & $0.518-0.944$ \\
\hline MO & 90 & 100 & & \\
\hline M1 & 0 & 0 & & \\
\hline
\end{tabular}

\section{Univariate and multivariate analyses}

The univariate analysis revealed that postoperative lymph node metastasis (two-field thoracic and abdominal lymph nodes), vascular tumor thrombus and tumor infiltration were associated with ESCC SCLN metastasis in elderly patients (positive) (Table 1). To identify predictors of SCLN metastasis, a multivariate analysis was performed using variables available before and during operations, including tumor location, preoperative CEA levels, postoperative lymph node metastasis (two-field thoracic and abdominal lymph nodes), vascular tumor thrombus and tumor 
Table 2 Logistic multivariate analysis of radical treatment for supraclavicular lymph node metastasis in elderly ESCC patients

\begin{tabular}{lcccc}
\hline Variable & $\beta$ & $\mathrm{P}$ & $\operatorname{Exp}(\mathrm{B})$ & $95.0 \% \mathrm{Cl}$ for $\operatorname{Exp}(\mathrm{B})$ \\
\hline Tumor thrombus & 3.379 & 0.002 & 29.339 & $3.198-269.183$ \\
Tumor infiltration & 2.112 & 0.003 & 8.252 & $2.155-31.585$ \\
Lymph node metastasis & 1.537 & 0.011 & 4.651 & $1.445-14.972$ \\
\hline
\end{tabular}

ESCC, esophageal squamous cell carcinoma.

infiltration. A logistic regression analysis revealed that postoperative lymph node metastasis $(\mathrm{P}=0.011)$, vascular tumor thrombus $(\mathrm{P}=0.002)$ and tumor infiltration $(\mathrm{P}=0.003)$ were independently associated with ESCC SCLN metastasis (Table 2).

\section{Recurrence and survival}

With a median follow-up time of 40 months, the 3 -year OS and disease-free survival (DFS) rates were $47.4 \%$ and $42.1 \%$ in SCLN-positive patients, respectively, which were significantly lower than the corresponding rates of $69.2 \%$ and $65.4 \%$ in SCLN-negative patients.

A Cox regression analysis revealed that postoperative lymph node metastasis (two-field thoracic and abdominal lymph nodes), vascular tumor thrombus and tumor infiltration were independently associated with SCLN metastasis (Table S1).

SCLN metastasis had a statistically significant impact on postoperative survival in elderly ESCC patients $(\mathrm{P}=0.028)$ which were showed in the Kaplan-Meier survival curve and cumulative risk curve. Over time, the cumulative risk of supraclavicular lymph node metastasis increased, the survival probability decreased, and the survival time was shortened (Figure 1) (Figures S1-S3).

\section{Nomogram and decision-making curve}

The points of each individual risk factor were combined on the top of the scale to determine the value of the corresponding factor and the total score was determined on the overall scale to predict supraclavicular lymph node metastasis in ESCC patients after surgery in terms of the probability (risk), survival probability and expected survival time; quantitative risk assessment of postoperative supraclavicular lymph node metastasis and survival prediction were applied for the individual risk assessment. This approach made the complex prediction more intuitive and easier to generalize and apply (Figures 2-4).

\section{Calibration curve}

The data is calculated by bootstrap resampling method to calculate the $\mathrm{C}$ index of the nomogram model to predict the survival rate is 0.806 (95\% CI: $0.738-0.874)>0.07$, indicating that the prediction accuracy and accuracy of the model are good. The calibration chart obtained by using bootstrap resampling to regroup the data and further incorporate it into the statistical program is shown in Figures 5,6. Through curve analysis, it is not difficult to see that the 3 - and 5-year survival probabilities predicted by the nomogram and actual observation results It is consistent. The prediction accuracy and accuracy are good and can be used to guide clinical decision-making.

\section{Decision curve}

By drawing the decision curve, it can be seen that PT value is in the range of $0.14-0.6$. The red curve is above the blue curve (also above the light grey and grey curves). In other words, when the risk of supraclavicular lymph node metastasis in elderly patients after radical resection of esophageal squamous cell carcinoma (ESCC) predicted by nomogram model is in the range of $0.14-0.63$, its independent risk factors can be used to predict and evaluate the survival probability of elderly patients with ESCC, which can be used to guide clinical decision-making (Figure 7).

\section{Discussion}

The clinical study of lymph node dissection in esophageal cancer began in the 1960s. The scope of (23) surgeries was extended from the middle and lower mediastinum and upper abdomen (traditional two-field dissection) (24-28) to the cervical and thoracic junction in the upper mediastinum (extended two-field dissection) (29) and then to the neck (three-field dissection) (30-34). The value of lymph node dissection is reflected by the following aspects. (I) The 

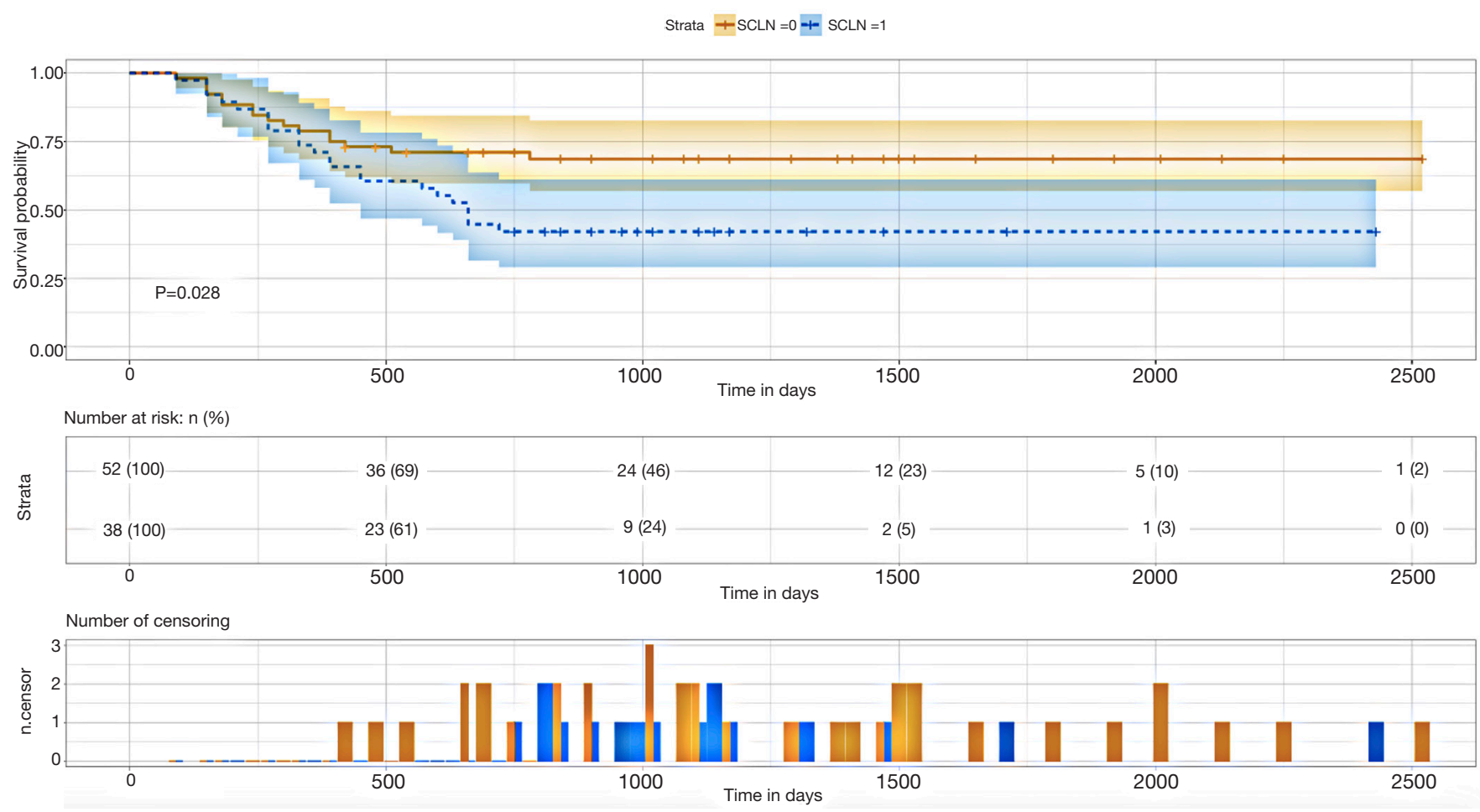

Figure 1 Kaplan-Meier and cumulative risk curves (effect of supraclavicular lymph node metastasis on postoperative survival in elderly ESCC patients and the cumulative risk) (log rank test). The above figure can be divided into three parts: upper, middle and lower. The $\mathrm{x}$-axis and $y$-axis represent the postoperative follow-up time and survival probability respectively [In yellow (SCLN =0, negative supraclavicular lymph node metastasis), the line represents the survival curve, and the width of the yellow bar represents the cumulative risk (the larger the width, the higher the cumulative risk).In blue (SCLN =1, positive supraclavicular lymph node metastasis), the line represents the survival curve, and the width of the blue bar represents the cumulative risk (the greater the width, the higher the cumulative risk). The $\mathrm{x}$-axis and $\mathrm{y}$-axis in the middle picture represent the number of survival cases and the postoperative follow-up time, respectively. The $x$-axis and $y$-axis in the figure below represent the density distribution of death population and the postoperative follow-up time. ESCC, esophageal squamous cell carcinoma; SCLN, supraclavicular lymph node.

accuracy of surgical pathological staging is improved. In particular, the 7th edition of the International Union Against Cancer (UICC) in 2009 proposed N classification according to the number of metastatic lymph nodes, so more than 12 lymph nodes must be dissected to ensure the accuracy of staging (35). (II) There is a deeper understanding of lymphatic metastasis in esophageal cancer. The lymph node groups with a high incidence of metastasis in thoracic esophageal cancer are the recurrent laryngeal nerve chain at the junction of the neck and thorax and the superior, middle and lower esophageal and cargo-gastric left artery-celiac artery chains. In terms of staging and radical treatment, systematic dissection of lymph nodes in these areas is required. (III) Radical surgery is improved, postoperative local recurrence is reduced, and long-term survival rates are improved. There has been a growing debate about the scope of lymph node dissection $(6,23-30,36)$. The reasonable selection of the range of lymph node dissection to ensure efficacy and reduce the negative effects of surgery is key. In particular, compared with young patients, elderly patients with ESCC have poor cardiopulmonary function reserve, relatively weak homeostasis and anti-strike ability of the internal environment, and they have a higher difficultly withstanding the trauma associated with surgery and postoperative complications.

The literature has reported that the prognosis of patients with supraclavicular metastasis after esophageal cancer surgery is poor, with a metastasis rate of approximately 10.3-17.6\%. Comprehensive treatment for patients with esophageal cancer with supraclavicular lymph node 


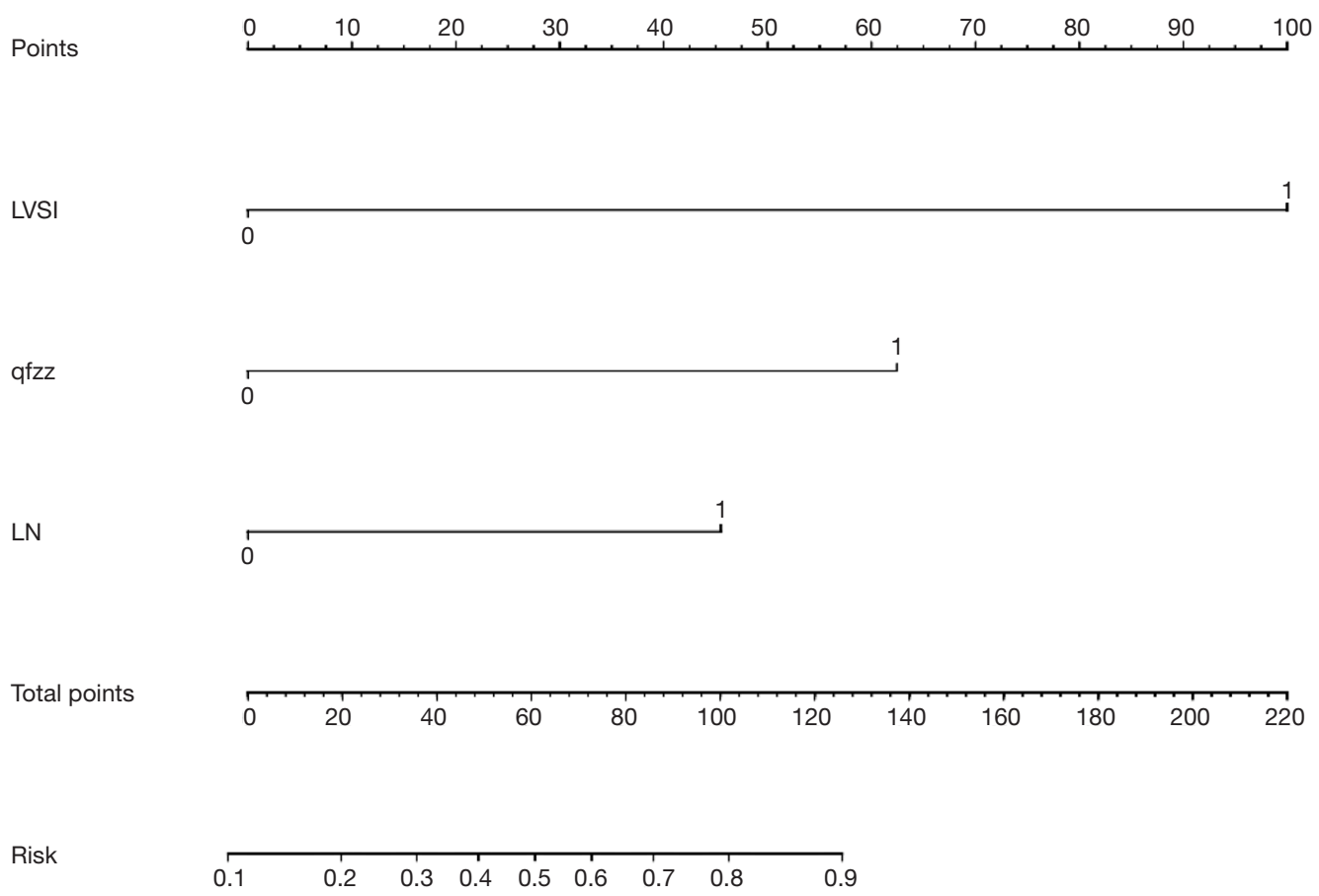

Figure 2 A nomogram of the risk of supraclavicular lymph node metastasis after radical resection of esophageal squamous cell carcinoma. To calculate the survival rate of each patient, first determine the score of each risk factor on the highest point scale, then add the scores of all risk factors to get the total score, and then project the value on Risk to indicate the risk of supraclavicular lymph node metastasis after radical resection of esophageal squamous cell carcinoma. LN, lymph node; qfzz, tumor infiltration; LVSI, vascular tumor thrombus.

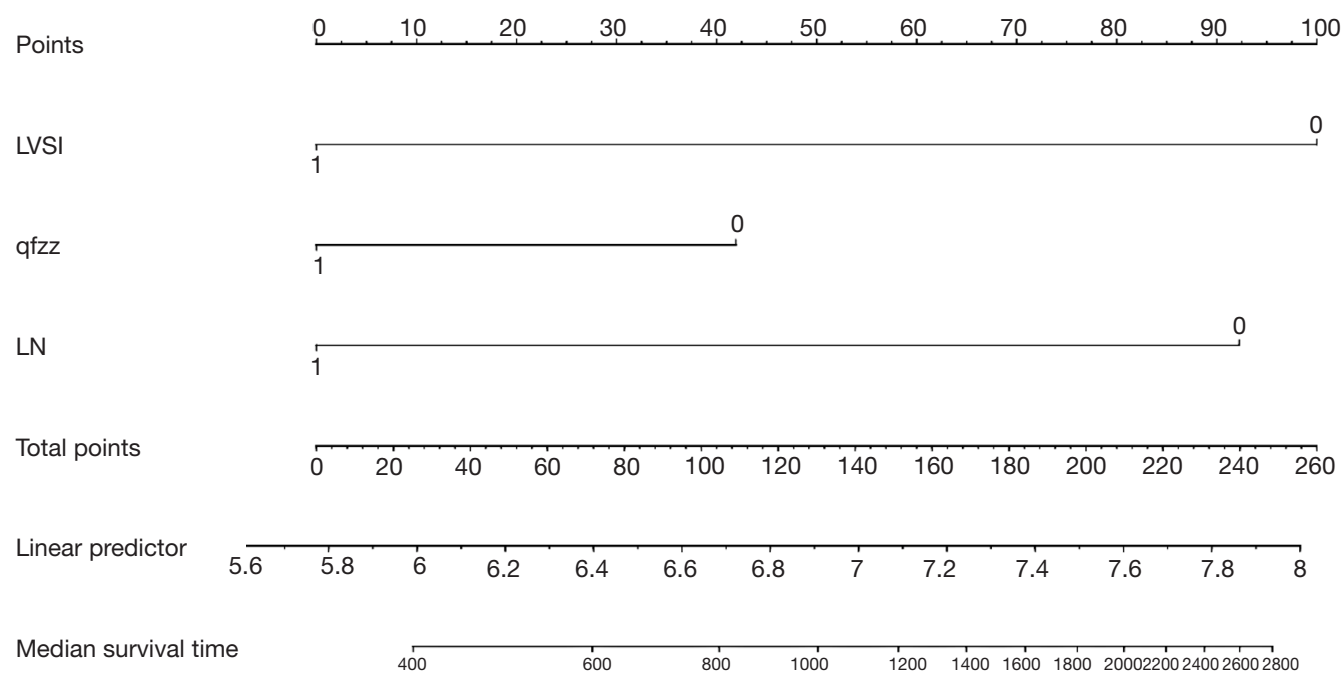

Figure 3 A nomogram of median survival time after radical resection of esophageal squamous cell carcinoma. To calculate the survival rate of each patient, first determine the score of each risk factor on the highest point scale, then add the scores of all risk factors to get the total score, and then project the value on the median survival time to indicate the median survival time. LN, lymph node; qfzz, tumor infiltration; LVSI, vascular tumor thrombus. 


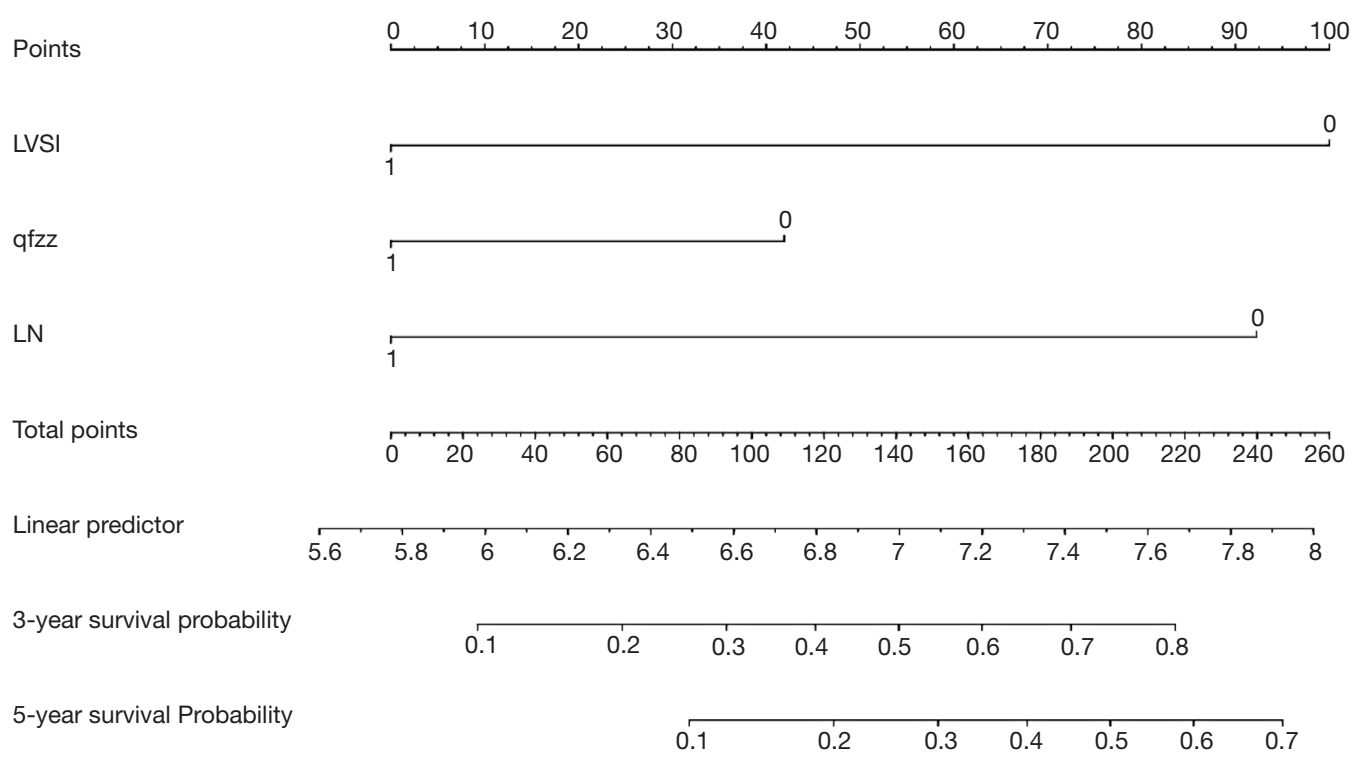

Figure 4 A nomogram of 3- and 5-year overall survival rates after radical resection of esophageal squamous cell carcinoma. To calculate the survival rate of each patient, first determine the score of each risk factor on the highest point scale, then add the scores of all risk factors to get the total score, and then project the value on the 3-year survival probability and 5-year survival probability to indicate the 3- and 5-year survival probability. LN, lymph node; qfzz, tumor infiltration; LVSI, vascular tumor thrombus.

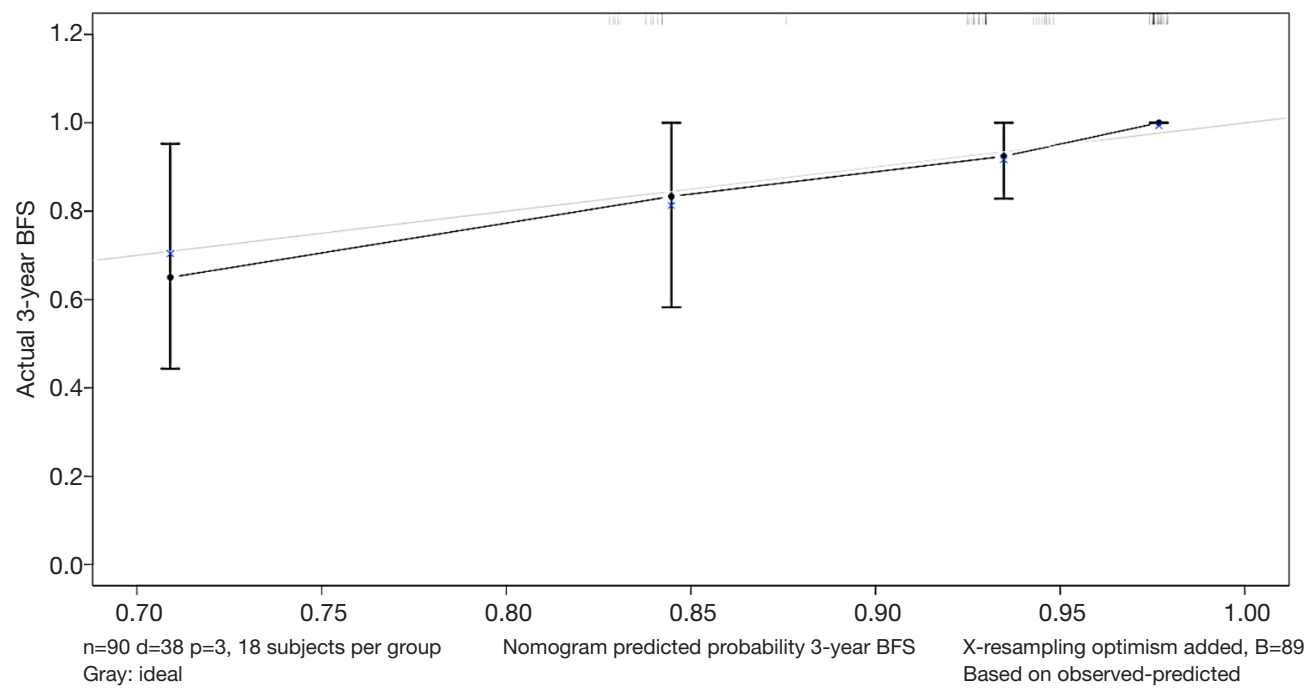

Figure 5 Calibration curve of nomogram model (3-year overall survival of this population). The $\mathrm{x}$-axis and $\mathrm{y}$-axis represent the predicted 3 -year survival probability and the actual survival probability of the nomogram model, respectively. 


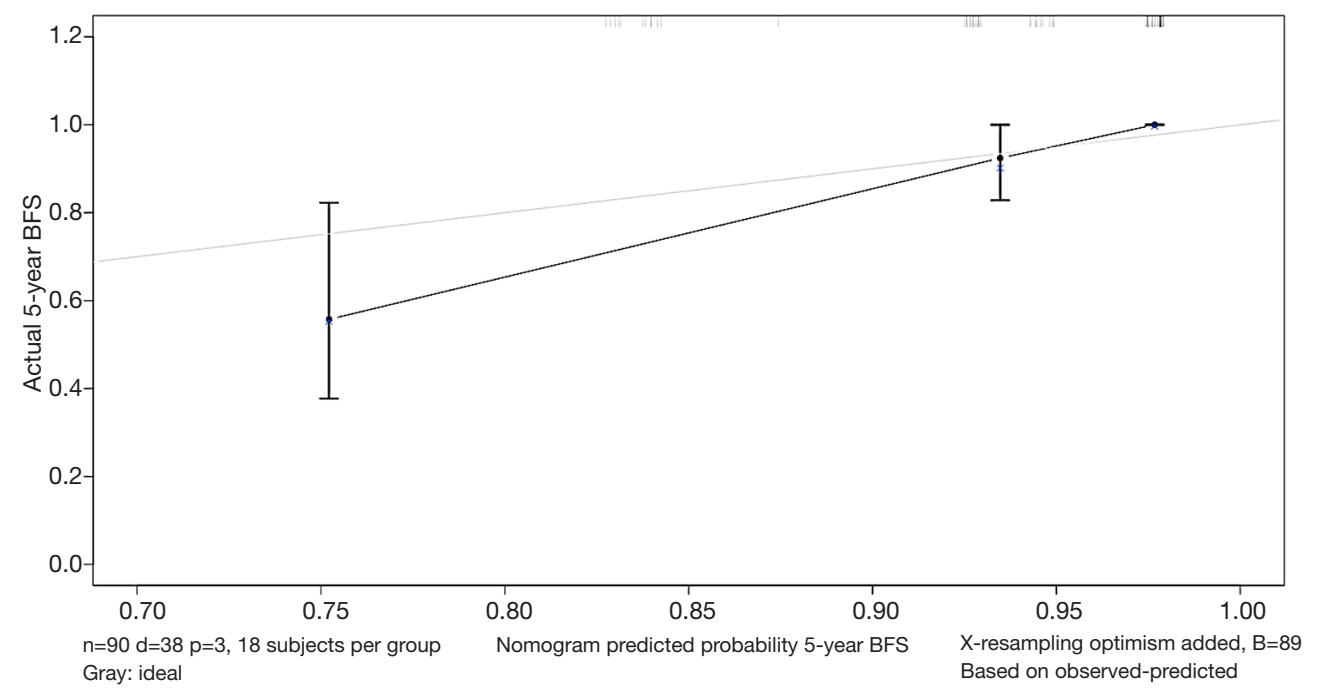

Figure 6 Calibration curve of nomogram model (5-year overall survival of this population). The $\mathrm{x}$-axis and $\mathrm{y}$-axis represent the predicted 5 -year survival probability and the actual survival probability of the nomogram model, respectively.

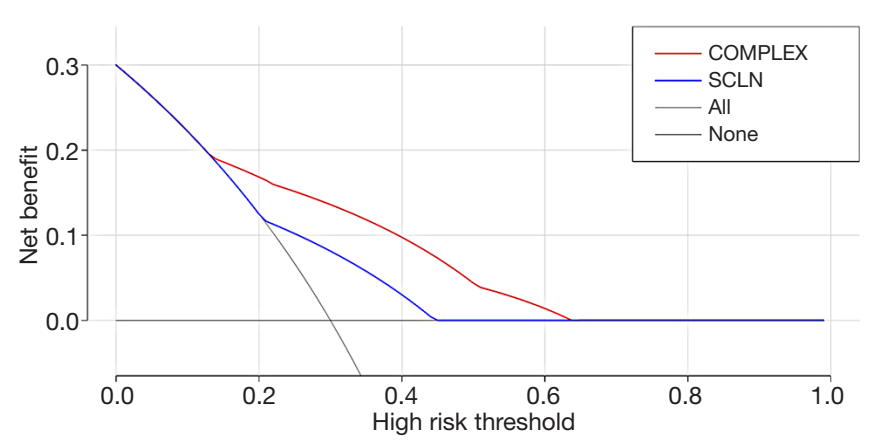

Figure 7 Decision curve. The $x$-axis in the figure represents the high-risk threshold. When various evaluation methods reach a certain value, the patient's metastasis probability is recorded as PI; when PI reaches a certain threshold (PT), it is defined as positive, and some intervention measures (such as supraclavicular lymph node dissection) are taken. The $y$-axis represents the net benefit (NB). [Red (complex): survival benefit of comprehensive prediction of vascular tumor thrombus, tumor invasion and postoperative lymph node metastasis; blue (SCLN): survival benefit of single factor prediction of supraclavicular lymph node; light gray (all): survival benefit of all supraclavicular lymph node dissection; dark gray (none): survival benefit without supraclavicular lymph node dissection]. SCLN, supraclavicular lymph node.

metastasis can significantly improve the survival rate of patients (37-41). In recent years, some studies on "selective lymph node dissection" have been carried out to conduct targeted dissection in patients with a high risk of cervical lymph node metastasis and to avoid the additional trauma caused by unnecessary expansion during surgery. For example, Professor Nakagawa et al. proposed neck dissection for upper thoracic tumors only (9). Professor Noguchi et al. attempted to introduce the concept of "sentinel lymph nodes", that is, cervical field dissection was only added in cases of intraoperative middle and upper mediastinal lymph node metastasis (10). All of the above results represent new directions to ensure radical surgery, reduce surgical risk and make treatment more reasonable. According to the anatomical and biological characteristics of lymph node metastasis in esophageal cancer, standard selection and reasonable dissection are key to improving the efficacy of esophageal cancer treatment.

Currently, how to use relevant clinical indicators to predict the risk of supraclavicular lymph node metastasis before the pathological diagnosis of positive supraclavicular lymph node metastasis, how to develop opportunities for treatment, and how to provide a basis for clinical decision-making (such as salvage chemoradiotherapy or supraclavicular lymph node dissection) are still unknown. By searching multiple databases, including PubMed, the nomogram model has been widely and successfully applied for the prediction and survival analysis of a variety of cancers. By considering all known clinical variables to quantify risk, the nomogram model allows objective and individualized risk assessment and prognosis prediction of a variety of cancers. To date, however, a related prediction model that can be applied for the clinical quantitative risk 
assessment of supraclavicular lymph node metastasis after ESCC surgery has not been established. Therefore, this article establishes a nomogram prediction model for the first time by analyzing the known clinical data to quantify the risk of postoperative supraclavicular lymph node metastasis in elderly patients with ESCC and to anticipate the prognosis for individual risk assessment guidance and decision making.

This paper analyzed the value of relevant clinical indicators for the risk assessment of supraclavicular lymph node metastasis after ESCC surgery in elderly patients, mainly because relevant indicators may have an important impact on the early diagnosis and intervention of supraclavicular lymph node metastasis after ESCC surgery in elderly patients. In relevant studies, some clinical indicators have been proven to be of great significance for evaluating the prognosis and diagnosis of cancer, especially vascular tumor thrombus. Several previous studies have shown that vascular tumor plugs are associated with the prognosis of various cancers (including ESCC) (42-45). In our study, vascular thromboembolism remained an independent prognostic risk factor. In this study, we attempted to establish a nomogram model based on postoperative clinical factors such as lymph node metastasis, vascular tumor thrombus, and invasive tissue to evaluate the risk of intraoperative supraclavicular lymph node metastasis in elderly ESCC patients and to guide clinical decisionmaking. Through analysis, the following conclusions can be made: (I) elderly patients with positive vascular tumor thrombus after ESCC surgery have a significantly increased risk of supraclavicular lymph node metastasis $(78 \% \leq$ risk); (II) we defined risk $>78 \%$ as high-risk patients, including patients with negative vascular tumor thrombotic and tumor infiltration tissues and postoperative lymph node metastasis (two-field thoracic and abdominal lymph nodes) (two-field thoracic and abdominal lymph nodes) (risk $=83 \%$ ); (III) even elderly ESCC patients with negative independent risk factors still have a $10 \%$ risk of supraclavicular lymph node metastasis, so follow-up observation is still needed for these patients after surgery, and we defined this group of patients as low-risk patients ( $10 \% \leq$ risk <39\%); (IV) we defined $78 \% \leq$ risk as high-risk population, $39 \% \leq$ risk $<78 \%$ as medium-risk patients, and $10 \%<$ risk $<39 \%$ as low-risk patients. We believe that our model is an easy tool for both physicians and patients to quantify the risk of postoperative supraclavicular lymph node metastasis. For example, a patient with negative lymph node metastasis [0], positive vascular thromboembolism [100], and noninvasive tissue [0] would obtain a total score of 100 , which translates to a $78 \%$ probability of supraclavicular lymph node metastasis. The patient was considered a highrisk patient, and it was not difficult to analyze the survival curve to find that supraclavicular lymph node metastasis had a statistically significant effect on the postoperative survival of elderly ESCC patients $(\mathrm{P}=0.028,<0.05)$. As time went on, the cumulative risk of supraclavicular lymph node metastasis increased, the survival probability decreased, and the survival time was shortened. Therefore, to reduce the risk of postoperative metastasis, improve the survival probability and prolong the survival time, we suggest that patients receive comprehensive treatment, including salvage chemoradiotherapy and supraclavicular lymph node dissection.

We must acknowledge several limitations of this study. First, the current study was a retrospective design of a small population. Potential biases were inevitable because of the retrospective nature of our study. Although some advanced statistical methods were applied to balance the covariates among the arms, there were still some latent biases. Secondly, the risk stratification of postoperative supraclavicular lymph node metastasis is not mature. Most importantly, this study only included single center samples to establish the nomogram model. In future further research, a more representative multi center sample of elderly patients with esophageal squamous cell carcinoma is needed to further improve the risk assessment value and survival benefit of the nomogram prediction model.

\section{Conclusions}

In conclusion, our results provide preliminary evidence that postoperative lymph node metastasis, vascular tumor thrombus, and tumor infiltration are independent risk factors for the development of supraclavicular lymph node metastasis in ESCC radical surgery based on the mathematical models of the column chart; these factors can be used to analyze risk assessment and survival in patients with indicators, as well as the risk of radical chest lymph node removal with twofield lymphadenectomy if further retrospective radiation and chemotherapy are needed postoperatively in patients with ESCC or supraclavicular lymph node cleaning treatment to provide a quantitative basis of risk.

\section{Acknowledgments}

Funding: The article was funded by Fujian Key Laboratory 
of Cardio-Thoracic Surgery (Fujian Medical University).

\section{Footnote}

Reporting Checklist: The authors have completed the TRIPOD reporting checklist. Available at http://dx.doi. org/10.21037/jtd-20-1388

Data Sharing Statement: Available at http://dx.doi. org/10.21037/jtd-20-1388

Peer Review File: Available at http://dx.doi.org/10.21037/jtd20-1388

Conflicts of Interest: All authors have completed the ICMJE uniform disclosure form (available at http://dx.doi. org/10.21037/jtd-20-1388). The authors have no conflicts of interest to declare.

Ethical Statement: The authors are accountable for all aspects of the work in ensuring that questions related to the accuracy or integrity of any part of the work are appropriately investigated and resolved. The study was conducted in accordance with the Declaration of Helsinki (as revised in 2013). The study was approved by institutional/ regional/national ethics/committee/ethics board of Fujian Union hospital (No.: 2020KY017) and informed consent was taken from all the patients.

Open Access Statement: This is an Open Access article distributed in accordance with the Creative Commons Attribution-NonCommercial-NoDerivs 4.0 International License (CC BY-NC-ND 4.0), which permits the noncommercial replication and distribution of the article with the strict proviso that no changes or edits are made and the original work is properly cited (including links to both the formal publication through the relevant DOI and the license). See: https://creativecommons.org/licenses/by-nc-nd/4.0/.

\section{References}

1. Chen W, Zheng R, Zhang S, et al. The incidences and mortalities of major cancers in China, 2009. Chin J Cancer 2013;32:106-12.

2. Chen W, Zheng R, Zhang S, et al. Report of incidence and mortality in China cancer registries, 2009. Chin J Cancer Res 2013;25:10-21.

3. Jemal A, Bray F, Center MM, et al. Global cancer statistics.
CA Cancer J Clin 2011;61:69-90.

4. Uzunoglu FG, Reeh M, Kutup A, et al. Surgery of esophageal cancer. Langenbecks Arch Surg 2013;398:189-93.

5. Higuchi K, Koizumi W, Tanabe S, et al. Current management of esophageal squamous-cell carcinoma in Japan and other countries. Gastrointest Cancer Res 2009;3:153-61.

6. Ma GW, Situ DR, Ma QL, et al. Three-field vs two-field lymph node dissection for esophageal cancer: a metaanalysis. World J Gastroenterol 2014;20:18022-30.

7. Ye T, Sun Y, Zhang Y, et al. Three-field or two field resection for thoracic esophageal cancer: a meta-analysis. Ann Thorac Surg 2013;96:1933-41.

8. Booka E, Takeuchi H, Nishi T, et al. The impact of postoperative complications on survivals after esophagectomy for esophageal cancer. Medicine 2015;94:e1369.

9. Nakagawa S, Nishimaki T, Kosugi S, et al. Cervical lymphadenectomy is beneficial for patients with carcinoma of the upper and mid-thoracic esophagus. Dis Esophagus 2003;16:4-8.

10. Noguchi T, Wada S, Takeno S, et al. Two-step three-field lymph node dissection is beneficial for thoracic esophageal carcinoma. Dis Esophagus 2004;17:27-31.

11. Graesslin O, Abdulkarim BS, Coutant C, et al. Nomogram to predict subsequent brain metastasis in patients with metastatic breast cancer. J Clin Oncol 2010;28:2032-7.

12. Rudloff U, Jacks LM, Goldberg JI, et al. Nomogram for predicting the risk of local recurrence after breastconserving surgery for ductal carcinoma in situ. J Clin Oncol 2010;28:3762-9.

13. Valentini V, van Stiphout RG, Lammering G, et al. Nomograms for predicting local recurrence, distant metastases, and overall survival for patients with locally advanced rectal cancer on the basis of European randomized clinical trials. J Clin Oncol 2011;29:3163-72.

14. Albert JM, Liu DD, Shen Y, et al. Nomogram to predict the benefit of radiation for older patients with breast cancer treated with conservative surgery. J Clin Oncol 2012;30:2837-43.

15. Han DS, Suh YS, Kong SH, et al. Nomogram predicting long-term survival after $\mathrm{d} 2$ gastrectomy for gastric cancer. J Clin Oncol 2012;30:3834-40.

16. van der Gaag NA, Kloek JJ, de Bakker JK, et al. Survival analysis and prognostic nomogram for patients undergoing resection of extrahepatic cholangiocarcinoma. Ann Oncol 2012;23:2642-9. 
17. Wang Y, Li J, Xia Y, et al. Prognostic nomogram for intrahepatic cho langiocarcinoma after partial hepatectomy. J Clin Oncol 2013;31:1188-95.

18. Yang L, Shen W, Sakamoto N, et al. Population-based study evaluating and predicting the probability of death resulting from thyroid cancer and other causes among patients with thyroid cancer. J Clin Oncol 2013;31:468-74.

19. Lagarde SM, Reitsma JB, de Castro SM, et al. Prognostic nomogram for patients undergoing esophagectomy for adenocarcinoma of the esophagus or gastro- esophageal junction. Br J Surg 2007;94:1361-8.

20. Lagarde SM, Reitsma JB, Ten Kate FJ, et al. Predicting individual survival after potentially curative esophagectomy for adenocarcinoma of the esophagus or gastroesophageal junction. Ann Surg 2008;248:1006-13.

21. World Health Day 2012: ageing and health: toolkit for event organizers. 2012;1:10-1.

22. Lewis I. The surgical treatment of carcinoma of the esophagus with special reference to a new operation for growths of the middle third. Br J Surg 1946;34:18-31.

23. Logan A. The surgical treatment of carcinoma of the esophagus and cardia. J Thorac Cardiovasc Surg 1963;46:150-61.

24. Nakayam K, Yanagisawa F, Nabeya K, et al. Concentrated preoperative irradiation therapy. Arch Surg 1963;87:1003-18.

25. Katsura S. Surgical indication of esophageal cancer, prognosis after surgery, and postoperative complaints and their treatments. Jpn J Thorac Cardiovasc Surg 1957;6:600-5.

26. Akakura I, Nakamura Y, Kakegawa T, et al. Surgery of carcinoma of the esophagus with preoperative radiation. Chest 1970;57:47-57.

27. Seo S. Esophageal surgery. J Jpn Surg Assoc 1933;33:1461-505.

28. Osawa T. Esophageal surgery. J Jpn Surg Assoc 1933;34:1319-590.

29. Kinoshita I, Ohashi I, Nakagawa K, et al. Lymph node metastasis in esophageal cancer; with special reference to upper mediastinum and measures for its treatment. Jpn J Gastroenetrol Surg 1976;9:424-30.

30. Sannohe Y, Hiratsuka R, Doki K. Lymph node metastases in cancer of the thoracic esophagus. Am J Surg 1981;141:216-8.

31. Kakegawa T, Yamana H, Fujita H. Cervical lymph node dissection during radical esophagectomy. Surg Diagn Treat 1986;28:523-8.
32. Isono K, Onoda S, Okuyama K, et al. The significance of cervical lymph nodes dissection in radical operation of esophageal cancer. Surg Diagn Treat 1986;28:529-35.

33. Muto T, Hasegawa M, Katayanagi N, et al. Appraisal of cervical lymph node dissection for thoracic esophageal carcinoma. Surg Diagn Treat 1986;28:536-40.

34. Tsurumaru M, Akiyama H, Ono Y, et al. The significance of cervical lymph nodes dissection in radical operation of esophageal cancer. Surg Diagn Treat 1986;28:555-60.

35. Edge SB, Compton CC. The American Joint Committee on Cancer: the 7th edition of the AJCC cancer staging manual and the future of TNM. Ann Surg Oncol 2010;17:1471-4.

36. Booka E, Takeuchi H, Nishi T, et al. The impact of postoperative complications on survivals after esophagectomy for esophageal cancer. Medicine 2015;94:e1369.

37. Mine S, Watanabe M, Kumagai K, et al. Oesophagectomy with or without supraclavicular lymphadenectomy after neoadjuvant treatment for squamous cell carcinoma of the esophagus. Br J Surg 2018;105:1793-8.

38. Okamura A, Watanabe M, Kozuki R, et al. Supraclavicular and celiac metastases in squamous cell carcinoma of the middle thoracic esophagus. Langenbecks Arch Surg 2018;403:977-84.

39. Miyata H, Yamasaki M, Miyazaki Y, et al. Clinical Importance of Supraclavicular Lymph Node Metastasis After Neoadjuvant Chemotherapy for Esophageal Squamous Cell Carcinoma. Ann Surg 2015;262:280-5.

40. Tachimori Y, Ozawa S, Numasaki H, et al. Supraclavicular node metastasis from thoracic esophageal carcinoma: A surgical series from a Japanese multi-institutional nationwide registry of esophageal cancer. J Thorac Cardiovasc Surg 2014;148:1224-9.

41. Bonvalot S, Bouvard N, Lothaire P, et al. Contribution of cervical ultrasound and ultrasound fine needle aspiration biopsy to the staging of thoracic esophageal carcinoma. Eur J Cancer 1996;32:0-895.

42. Yuan Z, Cao D, Yu M, et al. Uterine and Cervical Adenosarcoma: A Retrospective Study of Overall Oncologic Outcomes and Fertility Preservation in EarlyStage Disease. Oncologist 2019;24:e870-9.

43. Xu F, Ma J, Yi H, et al. Clinicopathological Aspects of Small Cell Neuroendocrine Carcinoma of the Uterine Cervix: A Multicenter Retrospective Study and MetaAnalysis. Cell Physiol Biochem 2018;50:1113-22.

44. Bendifallah S, Canlorbe G, Raimond E, et al. A clue 
towards improving the European Society of Medical Oncology risk group classification in apparent early stage endometrial cancer? Impact of lymphovascular space invasion. Br J Cancer 2014;110:2640-6.

Cite this article as: Chen $\mathrm{L}, \mathrm{Yu} \mathrm{S}$, Jiang $\mathrm{X}$, Kang $\mathrm{M}$. Supraclavicular lymph node metastasis in elderly patients undergoing esophageal squamous cell carcinoma radical surgery: construction of risk and prognostic predictive nomograms. J Thorac Dis 2021;13(1):18-30. doi: 10.21037/jtd20-1388
45. Sevin BU, Lu Y, Bloch DA, et al. Surgically defined prognostic parameters in patients with early cervical carcinoma: A multivariate survival tree analysis. Cancer 1996;78:1438-46. 


\section{Supplementary}

Table S1 Cox multivariate analysis of radical treatment for supraclavicular lymph node metastasis in elderly ESCC patients

$\begin{array}{lllll}\text { Variable } & \beta & p & \operatorname{Exp}(\mathrm{B}) & 95.0 \% \mathrm{Cl} \text { for } \operatorname{Exp}(\mathrm{B}) \\ \text { Vascular tumor thromboembolus } & 0.897 & 0.023 & 2.453 & 1.135-5.303 \\ \text { Tumor infiltration } & 0.834 & 0.022 & 2.303 & 1.127-4.707 \\ \text { Lymph node metastasis } & 1.153 & 0.008 & 3.169 & 1.350-7.440\end{array}$

Strata $+L \mathrm{LN}=0+-\mathrm{LN}=1$

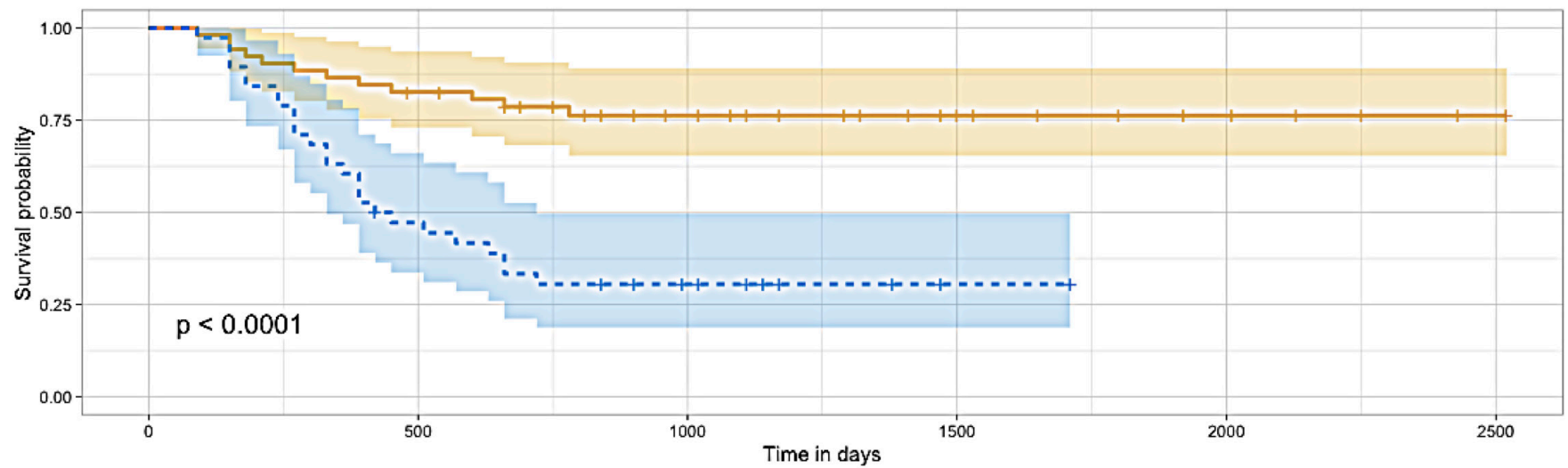

Number at risk: $\mathbf{n}(\%)$
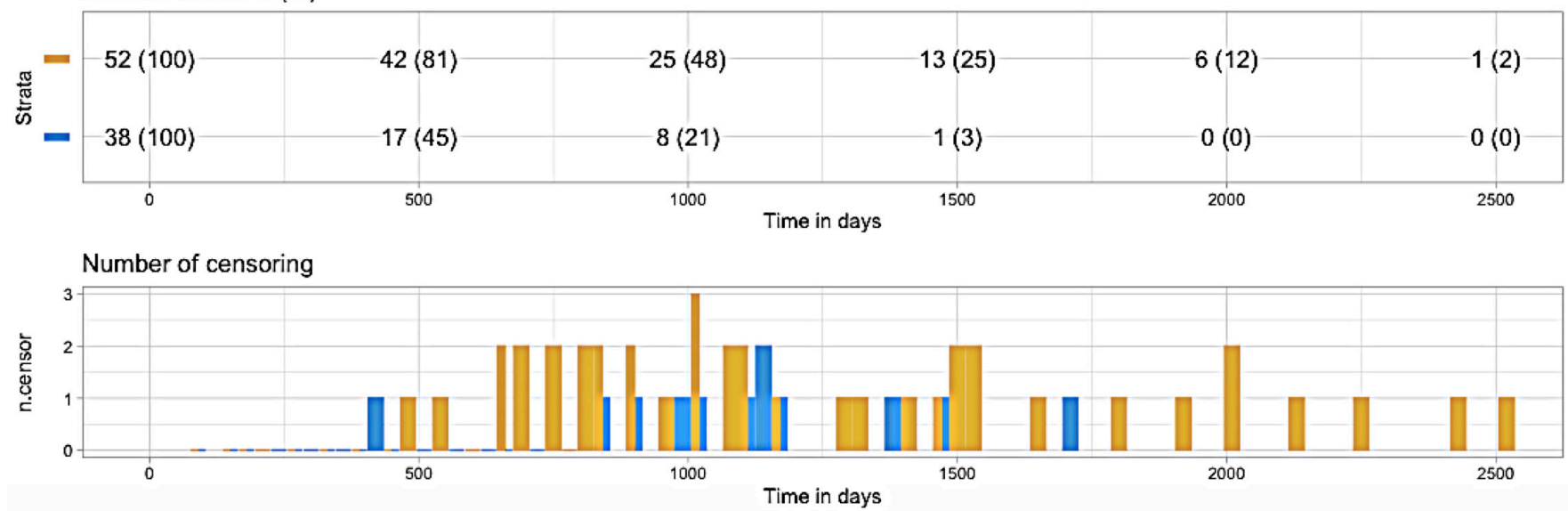

Figure S1 Kaplan-Meier and cumulative risk curves (effect of lymph node metastasis on postoperative survival in elderly ESCC patients and the cumulative risk) (log rank test). The above figure can be divided into three parts: upper, middle and lower. The $\mathrm{x}$-axis and $\mathrm{y}$-axis represent the postoperative follow-up time and survival probability respectively [In yellow ( $\mathrm{LN}=0$, negative lymph node metastasis), the line represents the survival curve, and the width of the yellow bar represents the cumulative risk (the larger the width, the higher the cumulative risk). In blue ( $\mathrm{LN}=1$, positive lymph node metastasis), the line represents the survival curve, and the width of the blue bar represents the cumulative risk (the greater the width, the higher the cumulative risk)]. The $\mathrm{x}$-axis and $\mathrm{y}$-axis in the middle picture represent the number of survival cases and the postoperative follow-up time, respectively. The $\mathrm{x}$-axis and $\mathrm{y}$-axis in the figure below represent the density distribution of death population and the postoperative follow-up time. 

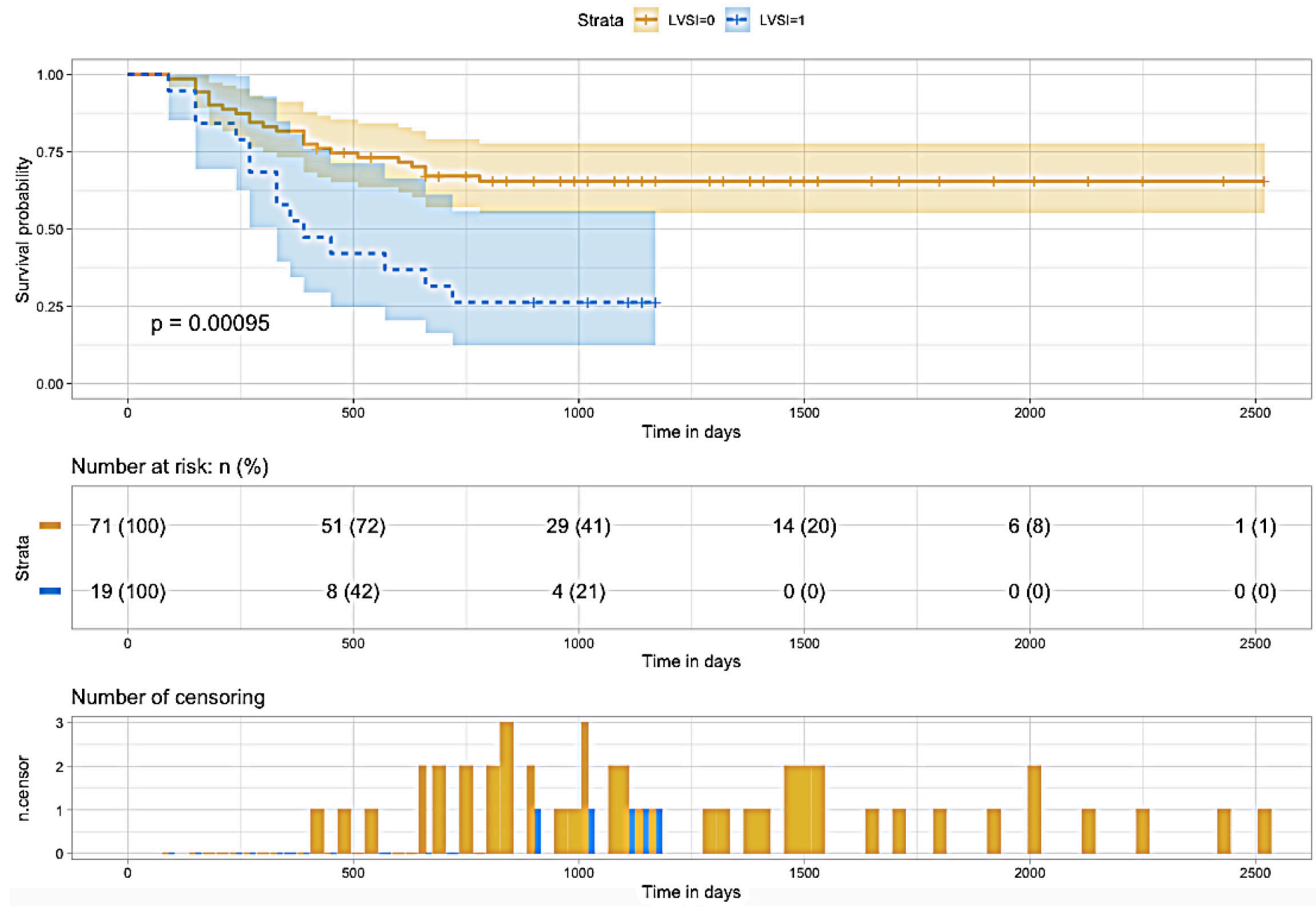

Figure S2 Kaplan-Meier and cumulative risk curves (effect of vascular tumor thrombus on postoperative survival in elderly ESCC patients and the cumulative risk) (log rank test). The above figure can be divided into three parts: upper, middle and lower. The $\mathrm{x}$-axis and $\mathrm{y}$-axis represent the postoperative follow-up time and survival probability respectively [In yellow (LVSI =0, negative thrombus), the line represents the survival curve, and the width of the yellow bar represents the cumulative risk (the larger the width, the higher the cumulative risk). In blue (LVSI =1, positive thrombus), the line represents the survival curve, and the width of the blue bar represents the cumulative risk (the greater the width, the higher the cumulative risk)]. The $\mathrm{x}$-axis and $\mathrm{y}$-axis in the middle picture represent the number of survival cases and the postoperative follow-up time, respectively. The $\mathrm{x}$-axis and $\mathrm{y}$-axis in the figure below represent the density distribution of death population and the postoperative follow-up time. 


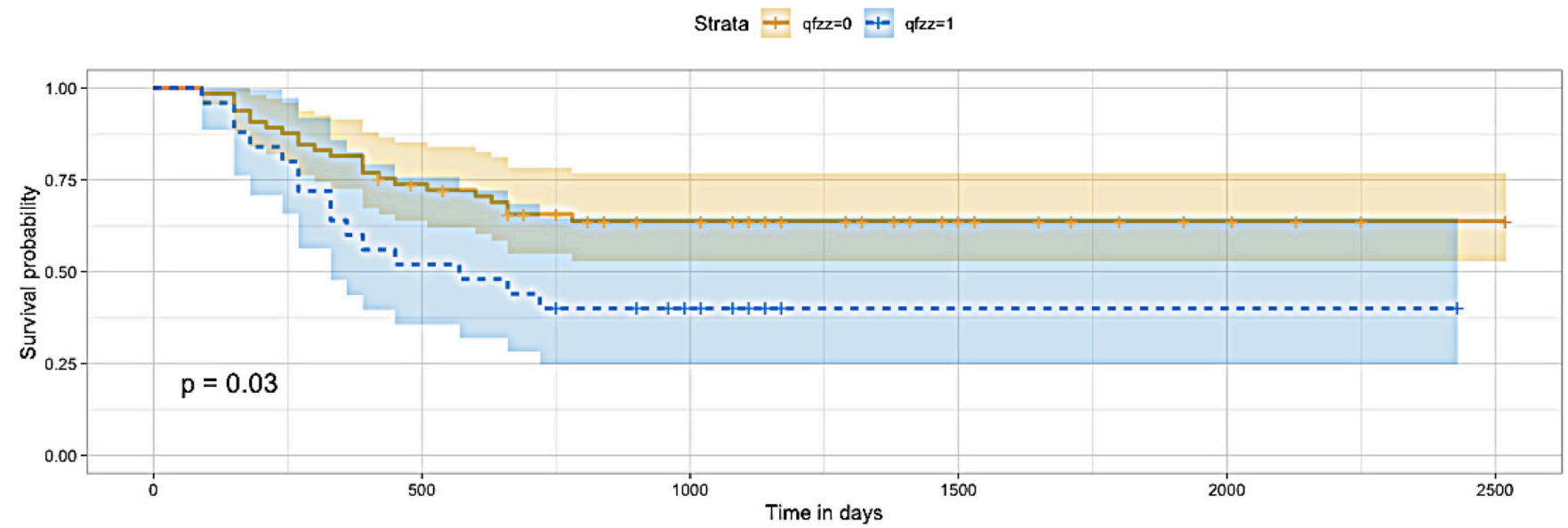

Number at risk: $\mathrm{n}(\%)$
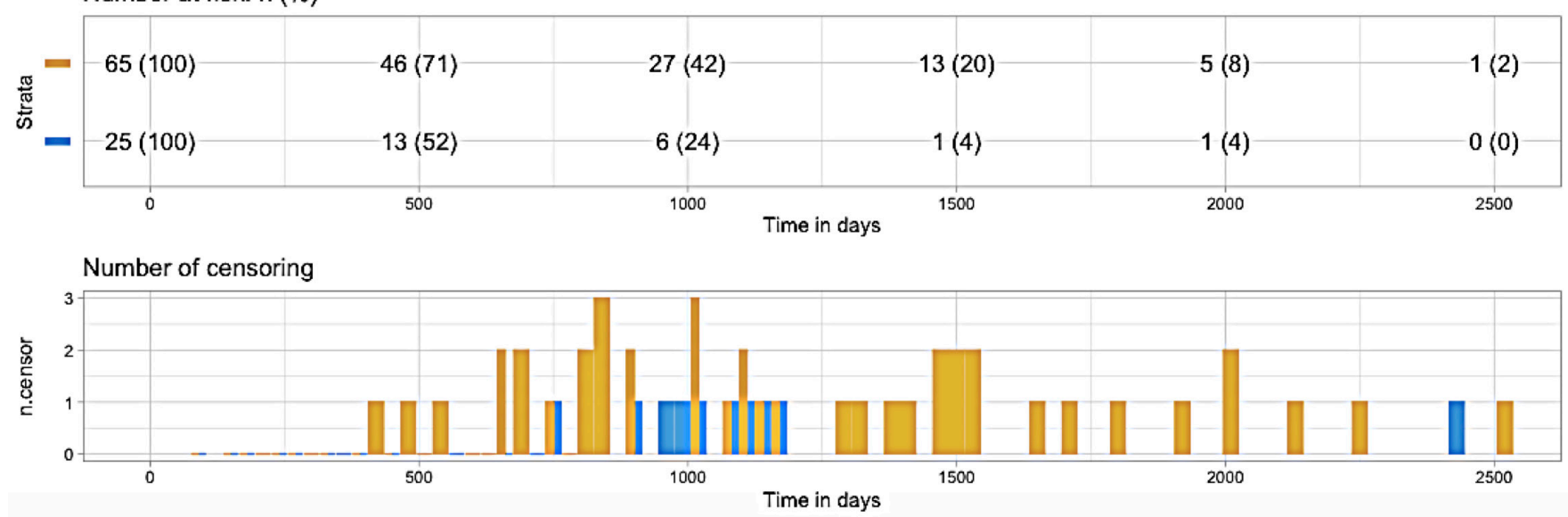

Figure S3 Kaplan-Meier and cumulative risk curves (effect of tumor infiltration on postoperative survival in elderly ESCC patients and the cumulative risk) (log rank test). The above figure can be divided into three parts: upper, middle and lower. The $\mathrm{x}$-axis and $\mathrm{y}$-axis represent the postoperative follow-up time and survival probability respectively [In yellow (qfzz =0, negative tumor infiltration), the line represents the survival curve, and the width of the yellow bar represents the cumulative risk (the larger the width, the higher the cumulative risk). In blue ( $\mathrm{qfzz}=1$, positive tumor infiltration), the line represents the survival curve, and the width of the blue bar represents the cumulative risk (the greater the width, the higher the cumulative risk)]. The $\mathrm{x}$-axis and $\mathrm{y}$-axis in the middle picture represent the number of survival cases and the postoperative follow-up time, respectively. The $\mathrm{x}$-axis and $\mathrm{y}$-axis in the figure below represent the density distribution of death population and the postoperative follow-up time. 